\title{
Effects of geometrical and material parameters on free vibration analysis of fiber metal laminated plates
}

\author{
Faramarz Ashenai Ghasemi ${ }^{1, a}$, Reza Paknejad ${ }^{1}$ and Keramat Malekzadeh Fard ${ }^{2}$ \\ 1 Department of Mechanical Engineering, Shahid Rajaee Teacher Training University (SRTTU), Lavizan, Postal \\ Code 16788-15811, Tehran, Iran \\ 2 Center of Excellence for Research in Advanced Materials and Structures, Department of Mechanical Engineering, \\ MUT University of Technology, Tehran, Iran
}

Received 22 February 2013, Accepted 22 May 2013

\begin{abstract}
Fiber metal laminates (FMLs) are high-performance hybrid structures based on alternating stacked arrangements of fiber-reinforced plastic (FRP) plies and metal alloy layers. In this paper, effect of some geometrical and material parameters on free vibrations of FML plates was studied. The first-order shear deformation theory (FSDT) as well as the Fourier series method was used to analytically solve the governing equations of the composite plate. The accuracy of the used method was verified by comparing the Rayleigh-Ritz analytical method and the ABAQUS finite element software (numerical) method. The results indicated that some of the important parameters like sequence of metal layers, aspect ratio $(a / b)$ of plate and orientation of composite fibers were important factors affecting free vibration of the FMLs.
\end{abstract}

Key words: Free vibration / fiber reinforced metal laminates (FMLs) / fiber reinforced plastic (FRP) / analytical method / first shear deformation theory

\section{Introduction}

Fiber metal laminates (FMLs) are hybrid structures consisting of different metal sheets and fiber-reinforced plastic (FRP) layers as shown in Figure 1. They combine good characteristics of metals such as ductility, impact and damage tolerances with the benefits of fiber composite materials such as high specific strength, high specific stiffness and good corrosion and fatigue resistance.

Due to the above advantages and also better lightweight characteristic of metals than FRP laminates, the plates composed of FML materials are excellent candidates for industries such as automobile, aircraft and space structures (Asundi and Choi [1], Vlot et al. [2] and Marsh [3]). Therefore, considering free vibration of the structure is necessary in the design process.

Many researchers have studied free vibration of composite plates using different methods. Ngo-Cong et al. [4] presented the free vibration analysis of laminated composite plates using FSDT and 1D-IRBFN method. The laminated composite plates with various boundary conditions, length-to-width ratios $(a / b)$, thickness-to-length ratios $(t / b)$ and material properties were considered.

\footnotetext{
a Corresponding author:

faramarz_ashenai_ghasemi@yahoo.com
}

Convergence study showed that faster rates were obtained for higher $t / b$ ratios irrespective of $a / b$ ratios of the rectangular plates. They also indicted that boundary conditions affected natural frequencies.

Xing and Liu [5] presented a novel separation of variables for solving the exact solutions for free vibrations of thin orthotropic rectangular plates using all combinations of simply supported and clamped boundary conditions.

Ganapathi et al. [6] calculated free vibrations' characteristics of simply supported anisotropic laminates using analytical approach. The formulation was based on the first-order shear deformation theory. The governing equations were obtained using energy method. They investigated effect of curvature, thickness ratio and lay up of the plate.

Leissa and Martin [7] studied free vibrations and buckling of composite plates with non-uniformly spaced fibers using Ritz method. Their numerical results for six non-uniform distributions of $E$-glass, graphite and boron fibers in epoxy matrices in simply supported square plates indicated that redistributions could increase buckling load and fundamental frequency of these structures.

Xing and Reddy [8] presented exact solution with Levy type solution method and a domain decomposition technique to investigate vibration behavior of rectangular 


\section{Nomenclature}

\begin{tabular}{|llll|}
\hline$a$ & Length of the plate & $E_{i j}$ & Young's modulus \\
$b$ & Width of the plate & $k_{\mathrm{sh}}$ & Shear correction factor \\
$h$ & Thickness of the plate & $\omega_{m n}$ & Natural frequency \\
$x, y, z$ & Coordinates & $v_{i j}$ & Poisson's ratio \\
$A_{i j}$ & Extensional stiffness matrix & $\rho_{h}$ & Density of each layer \\
$B_{i j}$ & Coupling stiffness matrix & $u^{0}$ & Plate displacement in $x$ direction \\
$D_{i j}$ & Bending stiffness matrix & $v^{0}$ & Plate displacement in $y$ direction \\
$\theta$ & Fiber orientation & $w^{0}$ & Plate displacement in $z$ direction \\
$\psi_{x}$ & Shear rotation in the $x$ direction & $I$ & Moment of inertia of the plate \\
$\psi_{y}$ & Shear rotation in the $y$ direction & $Q_{i j}$ & Reduced stiffness matrix \\
$q$ & Dynamic normal load & $\lambda$ & Non-dimensional frequency parameter \\
$\Phi_{m}(x)$ & Geometric boundary conditions in the $x$ direction & $\Psi_{n}(y)$ & Geometric boundary conditions in the $y$ direction \\
\hline
\end{tabular}
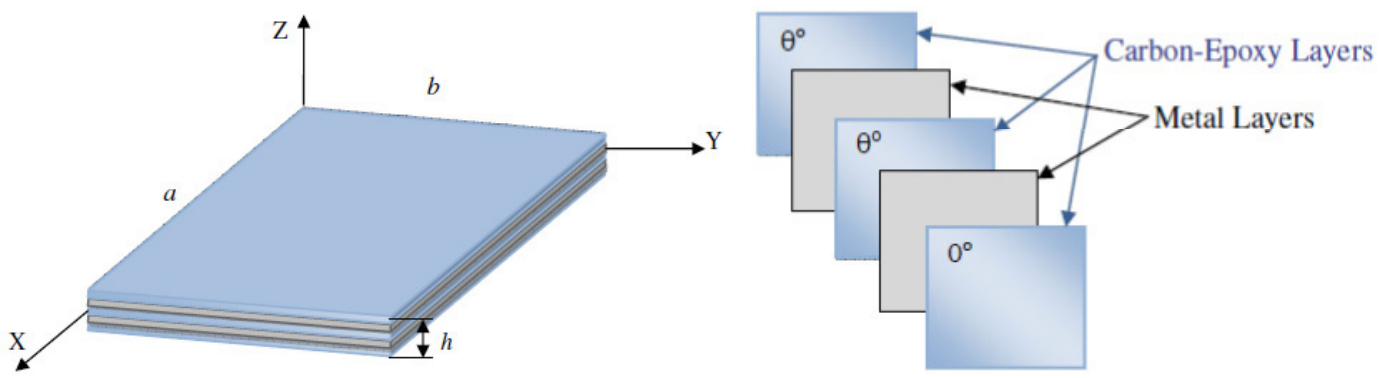

Fig. 1. Schematic view of fiber metal laminates (FMLs).

plates with an internal line hinge. They indicated that increase in the thickness ratio always decreased frequency parameters in plates.

Reddy and Kuppusamy [9] presented a description of the three-dimentional elasticity equations and the associated finite element model for natural vibrations of laminated rectangular plates. The numerical results were compared with those obtained by a shear deformable plate theory. Their investigations indicated that the twolayer angle-ply plates had higher frequencies than the cross-ply plates. These observations showed that the twolayer cross-ply laminates were structurally more flexible whereas the two-layer angle-ply laminates were stiffer than the four-layer cross-ply plates. They also indicated that the effect of the in-plane inertia was to reduce the frequencies, which especially had significant effects on higher modes.

Thai and Kim [10] studied the free vibration of laminated composite plates using two-variable refined plate theory (RPT), energy equations with Hamilton principle and Navier approach for simply support boundary condition. The Navier technique was employed to obtain closeform solutions of anti-symmetric cross-ply and angle-ply laminates. They showed that the refined plate theory was not only accurate but also efficient in predicting natural frequencies of anti-symmetric laminated composite plates. In order to avoid the use of shear correction factor in the first shear deformation theory (FSDT), Aagaah et al. [11] and Swaminathan and Patil [12] developed the higher-order shear deformation theories (HSDT) based on power series expansion of displacements with respect to the thickness coordinate. Using experiments and FEM numerical analysis, Mishra and Kumar Sahu [13] studied free vibration of woven fiber glass/epoxy composite plates in free-free boundary conditions. Their specimens of woven glass fiber and epoxy matrix composite plates were manufactured by the hand-layup technique. They also studied the effect of different geometrical parameters including number of layers, aspect ratio and fiber orientation of woven fiber composite plates for free-free boundary conditions in details. To examine effect of the number of layers of laminates, three different types of laminate were fabricated, which were made up of 8,12 and 16 layers. They indicated when the number of layers and aspect ratios increased, the natural frequency also increased. They also showed that, as the fiber angle increased, natural frequencies decreased. It was observed that the maximum frequency occurred at $\theta=0^{\circ}$ and the minimum at $\theta=30^{\circ}$.

Dutt and Shivanand [14] studied free vibration response of CFFF and CFCF woven carbon composite laminates using a Fast Fourier Transform analyzer and compared it with FEM tool ANSYS. Lee and Park [15] performed a free vibration analysis of skew composite laminates with or without cutout based on the high-order shear deformation plate theory (HSDT). They studied effects of skew angles and ply orientations on natural frequencies for various boundary conditions using a nonlinear high-order finite element program developed for this study. Civalek [16] investigated free vibration analysis of symmetrically laminated composite plates with first-order shear deformation theory (FSDT) by discrete 
singular convolution method. Thai and Kim [17] investigated free vibration of laminated composite plates using two-variable refined plate theory. Their theory accounted for parabolic distribution of transverse shear strains through plate thickness and satisfied the zero traction boundary conditions on the surface of the plate using shear correction factors. They used Hamilton's principle and the Navier technique to obtain the closed-form solutions of antisymmetric cross-ply and angle-ply laminates. Numerical results obtained using their theory were compared with those of three-dimensional elasticity solutions and those computed using the first-order and other higher-order theories. Hatami et al. [18] studied free vibration of axially moving symmetrically laminated plates subjected to in-plan force by classical plate theory. An exact method was developed to analyze vibration of multi-span traveling cross-ply laminates and then a semi-analytical finite strip method was extended for moving symmetric laminated plates in general for arbitrary boundary conditions.

Xiang and Wang [19] used trigonometric shear deformation theory to derive differential governing equations of symmetric laminated composite plates. Differential governing equations were discretized by a meshless collocation method based on the inverse multiquadric radial basis function to predict free vibration behavior of symmetric laminated composite plates. Natural frequencies were computed for various material parameters and geometry parameters of laminated plates and were compared with some available published results. The influence of grid pattern, modulus ratio and side-to-thickness ratio on natural frequencies was also investigated.

Topal and Uzman [20] investigated free vibration analysis of simply supported laminated composite plates using first-order shear deformation theory (FSDT). The displacement field of a laminated composite plate was given for FSDT. The numerical studies were conducted to determine effect of width-to-thickness ratio, degree of orthotropy and fiber orientation, aspect ratio on the non-dimensional fundamental frequency for laminated composite plates. Also, effect of shear deformation, rotary inertia and shear correction coefficients on the nondimensional fundamental frequency was examined.

Zhen and Wanji [21] used global-local higher-order theory to study free vibration of laminated composite and sandwich plates. This global-local theory could satisfy free surface conditions and geometric and stress continuity conditions at interfaces and the number of unknowns was independent from the layers number of the laminate. For general laminated composite plates, the results obtained from their global-local higher-order theory were found to be in good agreement with those obtained from threedimensional elasticity theories. They indicated that this theory was suitable for analyzing laminated plates with arbitrary layouts and soft-core sandwich plates whereas numerical results showed that the global higher-order and first-order theory overestimated natural frequency for these special structures. This theory could not only calculate natural frequencies but could accurately predict modal stress distributions in the thickness direction without any smooth techniques.

In this paper, free vibration of FMLs was investigated using two analytical and one numerical method. The effect of using Al layers as well as some of the important parameters such as stacking sequence, aspect ratio $(a / b)$ of the plate and orientation of composite medium fibers as important factors affecting free vibration of FMLs were studied as well. The results of these parameters could be useful for designers.

\section{Governing equations}

\subsection{First-order shear deformation theory}

Here, the plate equations developed by Whitney and Pagano [22] were used. They include effect of transverse shear deformations, in which displacement field of the FSDT at time is as follows:

$$
\begin{aligned}
u & =u^{0}(x, y, t)+z \psi_{x}(x, y, t) \\
v & =v^{0}(x, y, t)+z \psi_{y}(x, y, t) \\
w & =w^{0}(x, y, t)
\end{aligned}
$$

$u^{0}, v^{0}$ and $w^{0}$ are plate displacements in $x, y$ and $z$ directions at the plate mid-plane and $\psi_{x}$ and $\psi_{y}$ are shear rotations in $x$ and $y$ directions.

In this paper, a symmetrically laminated plate with the coordinate system originated at the mid-plane of laminate was considered, in which each layer of the laminate was orthotropic with respect to $x$ and $y$ directions and all layers were of equal thickness. Equations of motion for free vibration of this laminated plate could be expressed by dynamic version of the principle of virtual displacements as:

$$
\begin{gathered}
D_{11} \psi_{x, x x}+D_{66} \psi_{x, y y}+\left(D_{12}+D_{66}\right) \psi_{y, x y} \\
-k_{\mathrm{sh}} A_{55} \psi_{x}-k_{\mathrm{sh}} A_{55} w_{, x}=I_{2} \ddot{\psi}_{x} \\
\left(D_{12}+D_{66}\right) \psi_{x, x y}+D_{66} \psi_{y, x x}+D_{22} \psi_{y, y y} \\
-k_{\mathrm{sh}} A_{44} \psi_{y}-k_{\mathrm{sh}} A_{44} w_{, y}=I_{2} \ddot{\psi}_{y} \\
k_{\mathrm{sh}} A_{55} \psi_{x, x}+k_{\mathrm{sh}} A_{55} w_{, x x}+k_{\mathrm{sh}} A_{44} \psi_{y, y} \\
+k_{\mathrm{sh}} A_{44} w_{, y y}+q=I_{0} \ddot{w}
\end{gathered}
$$

$k_{\mathrm{sh}}$ is the shear correction factor introduced by Mindlin [23], which usually $\pi^{2} / 12$ and $q$ is the dynamic normal load over the plate; also:

$$
\begin{aligned}
\left(A_{i j}, B_{i j}, D_{i j}\right) & =\int_{-h / 2}^{h / 2} Q_{i j}^{k}\left(1, z, z^{2}\right) \mathrm{d} z \\
(\rho, I) & =\int_{-h / 2}^{h / 2} \rho_{0}\left(1, z^{2}\right) \mathrm{d} z
\end{aligned}
$$

where $I_{0}$ and $I_{2}$ are mass inertia tensor components defined as:

$$
\begin{aligned}
& I_{0}=\rho h \\
& I_{2}=\frac{\rho h^{3}}{12}
\end{aligned}
$$


in which $\rho_{0}$ represents the density of each layer, $\rho$ the total density and $h$ the thickness of the plate. $A_{i j}, B_{i j}, D_{i j}$ are the components of extensional and shear, coupling and bending stiffness matrices, respectively.

Whitney and Pagano [22] used $\left(Q_{i j}\right)_{k} \quad(i, j=$ $1,2,6)$ for reducing in-plane stiffness components and $\left(Q_{i j}\right)_{k}(i, j=4,5)$ for reducing transverse shear stiffness components. Expressing the variables $w, \psi_{x}, \psi_{y}$ in the following harmonic forms for the free vibration solution of a rectangular plate with simply supported boundary conditions was assumed to be in the following form $[24,25]$ :

$$
\begin{aligned}
w(x, y, t) & =\sum_{m=1}^{\infty} \sum_{n=1}^{\infty} A_{m n}(t)\left(\sin \frac{m \pi}{a} x\right)\left(\sin \frac{n \pi}{b} y\right) \\
\psi_{x}(x, y, t) & =\sum_{m=1}^{\infty} \sum_{n=1}^{\infty} A_{m n}(t)\left(\cos \frac{m \pi}{a} x\right)\left(\sin \frac{n \pi}{b} y\right) \\
\psi_{y}(x, y, t) & =\sum_{m=1}^{\infty} \sum_{n=1}^{\infty} B_{m n}(t)\left(\sin \frac{m \pi}{a} x\right)\left(\cos \frac{n \pi}{b} y\right)
\end{aligned}
$$

where $A_{m n}(t), B_{m n}(t)$ and $W_{m n}(t)$ are time-dependent coefficients.

In this work, rotary inertia effect was neglected following Mindlin [23]. Load function could be demonstrated by Christoforou and Swanson [24] and Carvalho and Soares [26] as follows:

$$
\begin{aligned}
q(x, y, z) & =\sum_{m} \sum_{n} Q_{m n}(t) \sin \left(\frac{m \pi}{a}\right) x \sin \left(\frac{n \pi}{b}\right) y \\
Q_{m n}(t) & =\frac{4 F(t)}{a b} \sin \left(\frac{m \pi}{a}\right) x \sin \left(\frac{n \pi}{b}\right) y
\end{aligned}
$$

where $Q_{m n}(t)$ are terms of the Fourier series, $F(t)$ is impact load, $a$ and $b$ are plate length and width, respectively. Hence, using equations (2) and (6), the system of equations (2) could be reduced to the following system of ordinary decouple differential equations:

$$
\begin{aligned}
{\left[\begin{array}{lll}
L_{11} & L_{12} & L_{13} \\
L_{12} & L_{22} & L_{23} \\
L_{13} & L_{23} & L_{33}
\end{array}\right]\left\{\left[\begin{array}{c}
A_{m n}(t) \\
B_{m n}(t) \\
W_{m n}(t)
\end{array}\right]\right\} } \\
\quad=\left\{\left[\begin{array}{c}
0 \\
0 \\
Q_{m n}(t)-\rho h \ddot{W}_{m n}(t)
\end{array}\right]\right\}
\end{aligned}
$$

where:

$$
\begin{aligned}
& L_{11}=D_{11}\left(\frac{m \pi}{a}\right)^{2}+D_{66}\left(\frac{n \pi}{b}\right)^{2} \\
& L_{12}=L_{21}=\left(D_{12}+D_{66}\right)\left(\frac{m \pi}{a}\right)\left(\frac{n \pi}{b}\right) \\
& L_{13}=L_{31}=k_{\mathrm{sh}} A_{55}\left(\frac{m \pi}{a}\right) \\
& L_{22}=D_{66}\left(\frac{m \pi}{a}\right)^{2}+D_{22}\left(\frac{n \pi}{b}\right)^{2}+k_{\mathrm{sh}} A_{55} \\
& L_{23}=L_{32}=k_{\mathrm{sh}} A_{44}\left(\frac{n \pi}{b}\right) \\
& L_{33}=k_{\mathrm{sh}} A_{55}\left(\frac{m \pi}{a}\right)^{2}+k_{\mathrm{sh}} A_{44}\left(\frac{n \pi}{b}\right)^{2}
\end{aligned}
$$

Following Christoforou and Swanson's [24] procedure and using changes of variables as shown below:

$$
\begin{aligned}
A_{m n}(t) & =K_{A} W_{m n}(t) ; \quad B_{m n}(t)=K_{B} W_{m n}(t) \\
K_{A} & =\frac{L_{12} L_{23}-L_{13} L_{22}}{L_{11} L_{22}-L_{12}^{2}} ; \quad K_{B}=\frac{L_{12} L_{13}-L_{11} L_{23}}{L_{11} L_{22}-L_{12}^{2}}
\end{aligned}
$$

Equation (9) can be simplified as follows:

$$
\ddot{W}_{m n}(t)+\omega_{m n}^{2} W_{m n}(t)=\frac{Q_{m n}(t)}{\rho h}
$$

where:

$$
\omega_{m n}^{2}=\frac{L_{13} K_{A}+L_{23} K_{B}+L_{33}}{\rho h}
$$

$\omega_{m n}^{2}$ is square of the fundamental frequencies of the plate.

If the values of $m=n=1,2,3, \ldots$ and $q=0$ are inserted in equations (14) and (2), respectively, values of natural frequencies for FMLs could be calculated. In the present research, a simply supported rectangular plate with dimensions of $a$ and $b$ was chosen to study, in which the boundary conditions were as follows:

$$
\begin{aligned}
& w=\psi_{x, x}=\psi_{y}=0 \quad \text { at } \quad x=0, a \\
& w=\psi_{y, y}=\psi_{x}=0 \quad \text { at } \quad y=0, b
\end{aligned}
$$

\subsection{Rayleigh-Ritz method}

For a symmetrically thin laminated composite plate, the governing differential equation is given by Liew [27] as follows:

$$
\begin{array}{r}
D_{11} \frac{\partial^{4} W}{\partial x^{4}}+4 D_{16} \frac{\partial^{4} W}{\partial x^{3} \partial y}+2\left(D_{12}+2 D_{66}\right) \frac{\partial^{4} W}{\partial x^{2} \partial y^{2}} \\
+4 D_{26} \frac{\partial^{4} W}{\partial x \partial y^{3}}+D_{22} \frac{\partial^{4} W}{\partial y^{4}}-\rho h \frac{\partial^{2} W}{\partial t^{2}}=0
\end{array}
$$

The governing energy functional for the plate is:

$$
F=V-T
$$

where:

$$
\begin{gathered}
V=\frac{1}{2} \int_{A}\left\{D_{11}\left(\frac{\partial^{2} W}{\partial x^{2}}\right)^{2}+2 D_{12}\left(\frac{\partial^{2} W}{\partial x^{2}}\right)\left(\frac{\partial^{2} W}{\partial y^{2}}\right)\right. \\
+D_{22}\left(\frac{\partial^{2} W}{\partial y^{2}}\right)^{2}+4 D_{16}\left(\frac{\partial^{2} W}{\partial x^{2}}\right)\left(\frac{\partial^{2} W}{\partial x \partial y}\right) \\
\left.+4 D_{26}\left(\frac{\partial^{2} W}{\partial y^{2}}\right)\left(\frac{\partial^{2} W}{\partial x \partial y}\right)+4 D_{66}\left(\frac{\partial^{2} W}{\partial x \partial y}\right)^{2}\right\} \mathrm{d} A \\
T=\frac{1}{2} \rho h \omega^{2} \int_{A} W^{2} \mathrm{~d} A
\end{gathered}
$$

in which $A$ is area of plate. 
If vibration is assumed to be a harmonic motion, the solution of equation (16) may be written as:

$$
w(x, y, t)=W(x, y) \sin \omega t
$$

The Ritz method in solving equation (16) was applied by Wong [28], Meirovitch [29] and Bhat [30]. The following series was used to represent the deflection $(W(x, y))$ :

$$
W(x, y)=\sum_{m} \sum_{n} A_{m n} \Phi_{m}(x) \Psi_{n}(y)
$$

where $\Phi_{m}(x)$ and $\Psi_{n}(y)$ are appropriate functions which satisfy at least the geometric boundary conditions in the $x$ and $y$ directions, respectively.

Substitution of the deflection function $W(x, y)$ in equation (21) into the kinetic and strain energy equations (18) and (19) and minimization of Rayleigh quotient with respect to the coefficients $A_{m n}$ led to the eigenvalue equation represented by Bhat [30]:

$$
\begin{aligned}
& \sum_{m} \sum_{n}\left(C_{m n i j}-\lambda^{2}\left(E_{m i}^{(0,0)} \cdot F_{n j}^{(0,0)}\right)\right) A_{m n}=0 \\
& C_{m n i j}=D_{11} E_{m i}^{(2,2)} F_{n j}^{(0,0)}+\alpha^{4} D_{22} E_{m i}^{(0,0)} F_{n j}^{(2,2)} \\
& +\alpha^{2} D_{12}\left(E_{m i}^{(0,2)} F_{n j}^{(2,0)}+E_{m i}^{(2,0)} F_{n j}^{(0,2)}\right) \\
& +2 \alpha D_{16}\left(E_{m i}^{(2,1)} F_{n j}^{(0,1)}+E_{m i}^{(1,2)} F_{n j}^{(1,0)}\right) \\
& +2 \alpha^{3} D_{26}\left(E_{m i}^{(0,1)} F_{n j}^{(2,1)}+E_{m i}^{(1,0)} F_{n j}^{(1,2)}\right) \\
& +4 \alpha^{2} D_{66} E_{m i}^{(1,1)} F_{n j}^{(1,1)} \\
& E_{m i}^{(0,0)}=\int_{0}^{1}\left(\frac{d^{0} \Phi_{m}}{d x^{0}}\right)\left(\frac{d^{0} \Phi_{i}}{d x^{0}}\right) \mathrm{d} x \\
& =\int_{0}^{1}\left(\Phi_{m} * \Phi_{i}\right) \mathrm{d} x \\
& F_{n j}^{(1,1)}=\int_{0}^{1}\left(\frac{d^{1} \Psi_{n}}{d x^{1}}\right)\left(\frac{d^{1} \Psi_{j}}{d x^{1}}\right) \mathrm{d} y \\
& =\int_{0}^{1}\left(\frac{d \Psi_{n}}{d y}\right)\left(\frac{d \Psi_{j}}{d y}\right) \mathrm{d} y \\
& E_{m i}^{(r, s)}=\int_{0}^{1}\left(\frac{d^{r} \Phi_{m}}{d x^{r}}\right)\left(\frac{d^{s} \Phi_{i}}{d x^{s}}\right) \mathrm{d} x, \\
& F_{n j}^{(r, s)}=\int_{0}^{1}\left(\frac{d^{r} \Psi_{n}}{d y^{r}}\right)\left(\frac{d^{s} \Psi_{j}}{d y^{s}}\right) \mathrm{d} y \\
& E_{m i}^{(2,2)}=\int_{0}^{1}\left(\frac{d^{2} \Phi_{m}}{d x^{2}}\right)\left(\frac{d^{2} \Phi_{i}}{d x^{2}}\right) \mathrm{d} x, \\
& F_{n j}^{(2,2)}=\int_{0}^{1}\left(\frac{d^{2} \Psi_{n}}{d y^{r}}\right)\left(\frac{d^{2} \Psi_{j}}{d y^{s}}\right) \mathrm{d} y
\end{aligned}
$$$$
\lambda=\frac{\rho h \omega^{2} a^{4}}{D_{0}} ; D_{0}=\frac{E_{1} h^{3}}{12\left(1-v_{12} v_{21}\right)} ;
$$$$
\alpha=\frac{a}{b} ; \quad m, n, i, j=1,2,3, \ldots ; \quad r, s=0,1,2
$$

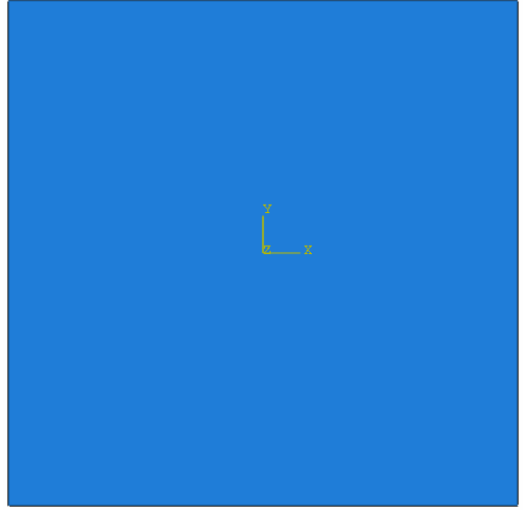

Fig. 2. Modeling in ABAQUS.

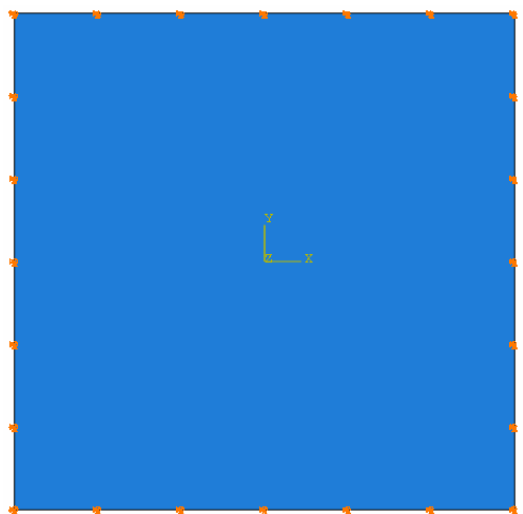

Fig. 3. Boundary condition in ABAQUS.

in which integrations as given in equation (24) are evaluated numerically. Natural frequencies and coefficients for the mode shapes equation (21) were obtained by solving the generalized eigenvalue problem defined by equation (22).

\subsection{Finite element (FE) model}

A three-dimensional finite element model was developed in the commercial software ABAQUS 6.11.3 for free vibrations of the composite and FML plates, as shown in Figure 2. This was done to carry out a comparative study for FE models with the analytical results. The material properties for this plate including S4R elements was used for meshing the simply support boundary condition of the composite plate (Fig. 3). The calculated frequencies were also listed in tables and compared with the analytical results. It can be seen that the calculated natural frequencies were close to the analytical values.

\section{Results and discussion}

The used FML plate was symmetric and cross-ply. The structure consisted of 10 layers, numbered from top to bottom. Material and geometrical properties of the FML plate are presented in Table 2 . 
Table 1. For a simply supported laminated composite plate having [0/90/0/90/0] layer sequence: the number of elements and nodes of the plate in ABAQUS.

\begin{tabular}{cccccccc}
\hline & \multicolumn{7}{c}{$a / b$} \\
\hline & 1 & 1.5 & 2 & 2.5 & 3 & 3.5 & 4 \\
\hline $\begin{array}{c}\text { Number of Elements } \\
\text { and Nodes }\end{array}$ & 61206 & 72360 & 78246 & 97686 & 117126 & 136566 & 156006 \\
\hline
\end{tabular}

Table 2. Geometrical and material properties of FML plate [31,32].

\begin{tabular}{c}
\hline Geometrical properties of FML plate: \\
Boundary conditions: Simply supported \\
Length $=$ Width $=200 \mathrm{~mm}$ \\
Lay-up: $[0 / 90 / 0 / 90 / 0]_{\mathrm{s}}$ \\
Ply thickness $=0.269 \mathrm{~mm}, a / h=74.3$ (Thin Plate) \\
\hline Material properties of composite plate $(\mathrm{T} 300 / 934$ carbon-epoxy layers): \\
$E_{11}=120 \mathrm{GPa} ; E_{22}=7.9 \mathrm{GPa} ; G_{12}=G_{13}=5.5 \mathrm{GPa}$ \\
$\nu_{12}=\nu_{23}=0.3 ; \rho=1580 \mathrm{~kg} \cdot \mathrm{m}^{-3}$ \\
\hline Material properties of Al layers $(2024-\mathrm{T} 3):$ \\
$E=72.4 \mathrm{GPa} ; \mathrm{G}=27.6 \mathrm{GPa} ; \nu=0.33 ; \rho=2780 \mathrm{~kg}^{-3} \mathrm{~m}^{-3}$
\end{tabular}

Table 3. For a simply supported laminated composite plate having $[0 / 90 / 0 / 90 / 0]_{s}$ layer sequence: effect of different solution methods and mode numbers on non-dimensional natural frequency $\left(\bar{\omega}=\left(\omega a^{2} / h\right) \sqrt{\rho / E_{1}}\right)$ for $)$ of the plate.

\begin{tabular}{ccccccc}
\hline \multicolumn{7}{c}{ Non-dimensional natural frequency $\left(\bar{\omega}=\left(\omega a^{2} / h\right) \sqrt{\rho / E_{1}}\right)$} \\
\hline Mode number \\
\hline Solution method & 1 & 2 & 3 & 4 & 5 & 6 \\
\hline FSDT & 3.2383 & 12.9023 & 28.8427 & 50.8203 & 78.5198 & 111.5663 \\
ABAQUS & 3.2306 & 12.8692 & 28.7616 & 50.6599 & 78.2411 & 111.4316 \\
Rayleigh-Ritz & 3.2425 & 12.9701 & 29.1829 & 51.8805 & 81.0628 & 116.7308 \\
Max. Error (\%) & 0.13 & 0.52 & 1.16 & 2 & 3.14 & 4.42 \\
\hline
\end{tabular}

Accuracy of the presented approach was verified analytically (by Rayleigh-Ritz method) and numerically (by ABAQUS software). Table 3 shows good agreement of the results. Table 3 shows that the maximum error of using the presented method was only about 4 percent. The effect of using Al layers and some of the parameters such as their stacking sequence, aspect ratio $(a / b)$ of the structure, orientation of composite medium fibers and increase in the thickness of the plate on the free vibration of FMLs is also studied in Table 3. By increasing the plate's aspect ratio, it tended to be converted into a rectangular strip and its lateral stiffness increased, this resulted in the increase of the effect of plate corners. Therefore, the plate showed a semi-cantilever behavior. This could be the reason that the results of the finite element method were different from those of analytical one.

\subsection{Effect of the layer sequence of the metal layers}

First, aluminum (Al) 2027-T3 layers were symmetrically placed instead of the carbon-epoxy T300/934 ones to see their position effects on the free vibration of the structure. It means that the Al layers were placed instead of these carbon-epoxy layers of the structure separately: $(1,10),(2,9),(3,8),(4,7),(5,6)$ and $(1,2,9,10)$, $(1,2,3,8,9,10),(1,2,3,4,7,8,9,10)$ and $(1,2,3,4,5,6,7,8,9,10)$.
Table 4 shows effect of different aspect ratios $(a / b)$ and layer sequences of FMLs on the first non-dimensional natural frequency using FSDT method in ABAQUS software. It can be seen that accuracy of the current analytical method results was verified by the numerical one. It was also observed that these values increased by using the Al layers instead of the carbon-epoxy layers and the aspect ratio $(a / b)$ of the plate also increased. Considering the material properties presented in Table 1 and because the lateral elastic modulus of the Al layers was greater than those of carbon-epoxy layers, with increase in the number of the $\mathrm{Al}$ layers, the amount of the natural frequency increased.

\subsection{Effect of the aspect ratio $(a / b)$ and location of the Al layers}

Table 5 shows effect of plate aspect ratio $(a / b)$ and also location of Al layers on natural frequency of the plate simultaneously. The results showed that, by increasing the aspect ratio and converting the plate into a strip, the non-dimensional natural frequency increased. Thus, the more the Al layers were embedded in the outer layers of the structure, the more the natural frequency would increase. This was because lateral stiffness of the Al layers was more than the FRP ones and, by embedding them in outer layers of the structure, their effects on the natural 
Table 4. For a simply supported laminated composite plate having $[0 / 90 / 0 / 90 / 0]_{\mathrm{s}}$ layer sequence: effect of different length-towidth $(a / b)$ ratios, solution methods and layer sequences on the first non-dimensional natural frequency $\left(\bar{\omega}=\left(\omega a^{2} / h\right) \sqrt{\rho / E_{1}}\right)$ of the plate.

\begin{tabular}{|c|c|c|c|c|c|c|c|c|}
\hline & & \multicolumn{7}{|c|}{ Non-dimensional natural frequency $\left(\bar{\omega}=\left(\omega a^{2} / h\right) \sqrt{\rho / E_{1}}\right)$} \\
\hline & & & & & $a / b$ & & & \\
\hline Layers sequence & Solution method & 1 & 1.5 & 2 & 2.5 & 3 & 3.5 & 4 \\
\hline No & FSDT & 3.2383 & 5.0819 & 8.0116 & 11.9358 & 16.8041 & 22.5938 & 29.2940 \\
\hline Al layers & ABAQUS & 3.2306 & 5.0728 & 8.0028 & 11.9275 & 16.7974 & 22.5899 & 29.2960 \\
\hline$(1,10)$ & FSDT & 4.1853 & 7.1396 & 11.3990 & 16.9274 & 23.7082 & 31.7340 & 41.0013 \\
\hline Al layers & ABAQUS & 4.1498 & 7.0999 & 11.3576 & 16.8851 & 23.6648 & 31.6919 & 40.9618 \\
\hline$(1,2,9,10)$ & FSDT & 4.8207 & 7.7550 & 11.9183 & 17.2978 & 23.8859 & 31.6788 & 40.6747 \\
\hline Al layers & ABAQUS & 4.7665 & 7.6920 & 11.8516 & 17.2295 & 23.8159 & 31.6102 & 40.6089 \\
\hline$(1,2,3,8,9,10)$ & FSDT & 5.2889 & 8.6628 & 13.3991 & 19.4946 & 26.9477 & 35.7574 & 45.9234 \\
\hline Al layers & ABAQUS & 5.2286 & 8.5940 & 13.3265 & 19.4196 & 26.8707 & 35.6810 & 45.8498 \\
\hline$(1,2,3,4,7,8,9,10)$ & FSDT & 5.6726 & 9.2083 & 14.1558 & 20.5157 & 28.2885 & 37.4741 & 48.0728 \\
\hline Al layers & ABAQUS & 5.6194 & 9.1471 & 14.0918 & 20.4514 & 28.2241 & 37.4145 & 48.0178 \\
\hline$(1,2,3,4,5,6,7,8,9,10)$ & FSDT & 6.0473 & 9.8250 & 15.1086 & 21.8996 & 30.1985 & 40.0058 & 51.3215 \\
\hline Al layers & ABAQUS & 6.0143 & 9.7874 & 15.0711 & 21.8643 & 30.1664 & 39.9811 & 51.3063 \\
\hline
\end{tabular}

Table 5. For a simply supported laminated composite plate with $[0 / 90 / 0 / 90 / 0]_{\mathrm{s}}$ layer sequence: effect of different lengthto-width $(a / b)$ ratios and location of Al layers on the first non-dimensional natural frequencies $\left(\bar{\omega}=\left(\omega a^{2} / h\right) \sqrt{\rho / E_{1}}\right)$ of the plate.

\begin{tabular}{cccccccc}
\hline & \multicolumn{7}{c}{$a / b$} \\
\hline Layer sequence & 1 & 1.5 & 2 & 2.5 & 3 & 3.5 & 4 \\
\hline No Al layers & 3.2383 & 5.0819 & 8.0116 & 11.9358 & 16.8041 & 22.5938 & 29.2940 \\
Al layers in (1,10) & 4.1853 & 7.1396 & 11.3990 & 16.9274 & 23.7082 & 31.4340 & 40.0013 \\
Al layers in (2,9) & 3.8872 & 5.6593 & 9.3407 & 14.8993 & 20.3073 & 28.5489 & 37.6156 \\
Al layers in $(3,8)$ & 3.6481 & 5.5338 & 9.0084 & 13.9959 & 19.6580 & 26.3773 & 34.1458 \\
Al layers in (4,7) & 3.4792 & 5.3685 & 8.7504 & 12.7426 & 17.6965 & 24.1891 & 31.0092 \\
Al layers in (5,6) & 3.3917 & 5.3337 & 8.4115 & 12.4302 & 17.3383 & 23.8122 & 30.7409 \\
\hline
\end{tabular}

frequency of the structure increased. Another reason was that the issue of high density of Al layers with respect to FRP ones, which resulted in the increase at moment of inertia in the plate. Therefore, the strength and stiffness of the plate and also natural frequency of the plate increased with respect to the cases that the Al layers were embedded in the middle layers of the structures.

\subsection{Effect of the fiber orientation of the composite plate}

Effect of using different fiber orientations of the composite plate was studied here (Tab. 6). To study effect of fiber orientations of the composite and the FML plate, the layup was chosen as $[0 / \theta / 0 / \theta / 0]_{\mathrm{S}}$ where $\theta=0^{\circ}, 15^{\circ}$, $30^{\circ}, 45^{\circ}, 60^{\circ}, 75^{\circ}, 90^{\circ}$. As can be seen in small values of $\theta$ (from $0^{\circ}$ to $45^{\circ}$ ), the first non-dimensional natural frequency of plate increased by increasing $\theta$. But, by increasing the amount of $\theta$ from $60^{\circ}$ to $90^{\circ}$, the first non-dimensional natural frequency of the plate decreased, which was a good advantage for designers of composite and FML structures in designing the stacking sequence of their desired structure.

It must be also noted that, with increasing the mode number, the amount of frequency also increased that was obvious. The closer the angle of the fibers to 45 degrees, shear and lateral stiffness of the plate would increase and therefore the natural frequency would increase (Tab. 6). Comparison of Ritz and finite elements method showed good accordance of the results for different fiber orientations. Because the aspect ratio $(a / b)$ of the plate was equal to one (with symmetrical geometry), the natural frequency of the plate increased from 0 to 45 degrees and decreased from 45 to 90 degrees. For the above reasons and also since shear stiffness was high in 45 degrees, natural frequency had its maximum value with respect to other fiber orientations (Tab. 6).

Figure 4 shows effect of aspect ratio and fibers' orientation on the non-dimensional natural frequency. When the aspect ratio was equal to one, the plate had a square shape. Therefore, because of the geometrical symmetry and the plate's boundary conditions, natural frequency increased when the fibers' orientation increased from 0 to 45 degrees and then decreased when they increased from 45 to 90 degrees. This process changed when aspect ratio increased and the plate tended to become a strip. For example, when the fibers' orientation was 90 degrees, the more the aspect ratio increased, the more the opposite sides of the plate got close to each other. Because the fibers' orientation was rectangular to these edges, the fibers tended similar to the small parallel beams. Therefore, the plate stiffness increased severely and became higher than other cases. When the fibers' orientation was 
Table 6. For a simply supported laminated composite plate with $[0 / 90 / 0 / 90 / 0]_{\mathrm{s}}$ layer sequence: effect of different fiber orientations $(\theta)$, mode numbers and solution methods on the non-dimensional natural frequencies $\left(\bar{\omega}=\left(\omega a^{2} / h\right) \sqrt{\rho / E_{1}}\right)$ of the plate.

\begin{tabular}{cccccccc}
\hline & \multicolumn{7}{c}{ Non-dimensional natural frequency $\left(\bar{\omega}=\left(\omega a^{2} / h\right) \sqrt{\rho / E_{1}}\right)$} \\
\hline & \multicolumn{7}{c}{ Mode number } \\
\hline$\theta^{\circ}$ & Solution method & 1 & 2 & 3 & 4 & 5 & 6 \\
\hline \multirow{2}{*}{0} & FSDT & 3.2363 & 12.8715 & 28.6910 & 50.3582 & 77.4423 & 109.4503 \\
& ABAQUS & 3.2288 & 12.8392 & 28.6105 & 50.1999 & 77.1669 & 109.3196 \\
\hline \multirow{2}{*}{15} & FSDT & 3.3284 & 13.2387 & 29.5124 & 51.8065 & 79.6816 & 112.6338 \\
& ABAQUS & 3.3016 & 13.1308 & 29.2473 & 51.2493 & 78.6871 & 110.6811 \\
\hline \multirow{2}{*}{30} & FSDT & 3.5054 & 13.9443 & 31.0910 & 54.5908 & 83.9873 & 118.7554 \\
& ABAQUS & 3.4410 & 13.7290 & 30.5799 & 53.6070 & 83.7366 & 116.3309 \\
\hline \multirow{2}{*}{45} & FSDT & 3.5909 & 14.2878 & 31.8697 & 55.9877 & 86.1911 & 121.9579 \\
& ABAQUS & 3.5057 & 14.1782 & 31.7570 & 55.0382 & 85.2257 & 120.3834 \\
\hline \multirow{2}{*}{60} & FSDT & 3.5063 & 13.9585 & 31.1608 & 54.8030 & 84.4809 & 119.7217 \\
& ABAQUS & 3.4365 & 13.1554 & 30.3481 & 53.9179 & 83.1641 & 119.4721 \\
\hline \multirow{2}{*}{75} & FSDT & 3.3301 & 13.2646 & 29.6399 & 52.1949 & 80.5865 & 114.4089 \\
& ABAQUS & 3.3001 & 13.0536 & 28.7027 & 51.6612 & 79.9798 & 113.7365 \\
\hline \multirow{2}{*}{90} & FSDT & 3.2383 & 12.9023 & 28.8427 & 50.8203 & 78.5198 & 111.5663 \\
& ABAQUS & 3.2306 & 12.8692 & 28.7616 & 50.6599 & 78.2411 & 111.4316 \\
\hline
\end{tabular}

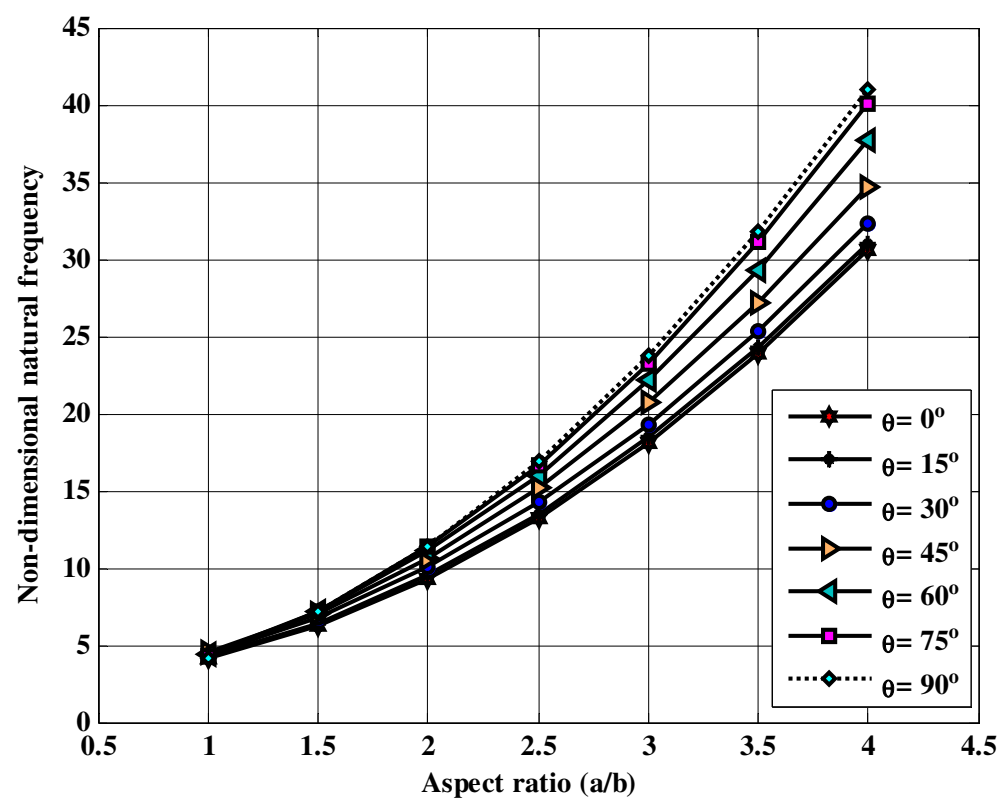

Fig. 4. For a simply supported laminated composite plate having $[0 / 90 / 0 / 90 / 0]_{\mathrm{s}}$ layer sequence with Al layers in $(1,10)$ plies: effect of the fiber orientations and length-to-width $(a / b)$ ratios on the first non-dimensional natural frequency $(\bar{\omega}=$ $\left.\left(\omega a^{2} / h\right) \sqrt{\rho / E_{1}}\right)$.

0 degree, this was vice versa. Therefore, depending on what the plate's aspect ratio or the fibers' orientation was, the plate's behavior changed. To study natural frequency of these structures, effect of this parameter must be simultaneously noted. This shows why the plate behavior is so complicated by changing the plate's aspect ratio and the fibers' orientation.

Figures 5 and 6 show the first and second mode shapes using analytical and FEM methods. A good adaptation could be observed between the results; the more the mode number increased, the mode shape became more complicated.

\section{Conclusions}

In the present research, free vibration of a simply supported laminated FML plate was studied using the firstshear deformation theory and Fourier series method to solve the system of governing equations of the plate analytically. The accuracy of the presented method was verified by comparing the data by the Rayleigh-Ritz (an analytical method) and the ABAQUS software (a numerical method). The results indicated that some parameters like layer sequence, aspect ratio and fibers' orientation of the plate were important factors which affected free vibration of the FMLs. Also, location of embedding the Al layers 


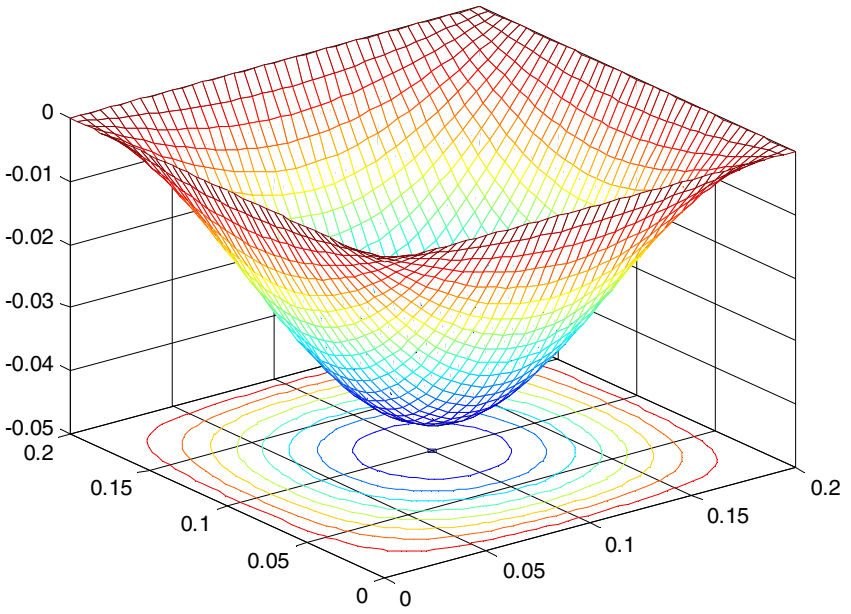

(a)

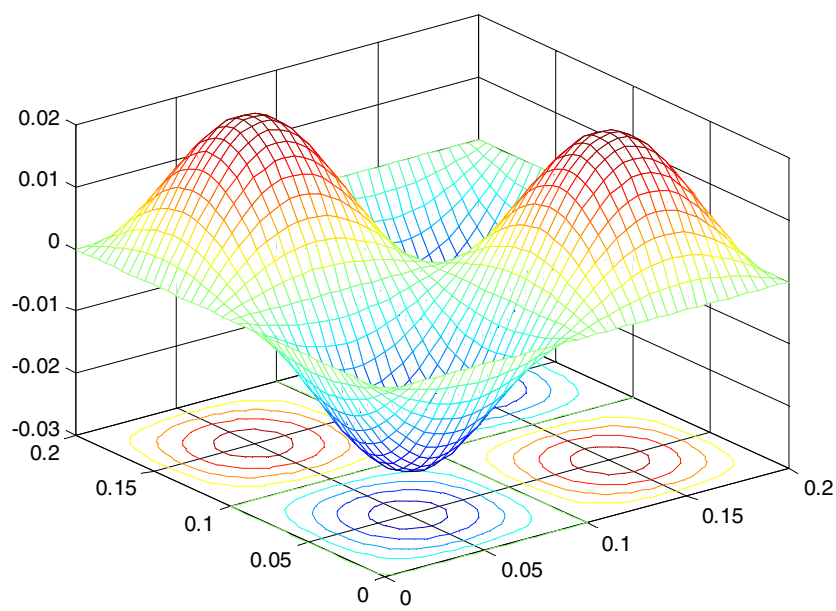

(b)

Fig. 5. Different mode shapes of a simply supported laminated composite plate with $[0 / 90 / 0 / 90 / 0]_{\mathrm{s}}$ layer sequence with $\mathrm{Al}$ layers in $(1,10)$ plies. (a) Mode 1: $\bar{\omega}=4.1853$. (b) Mode 2: $\bar{\omega}=16.6871$.

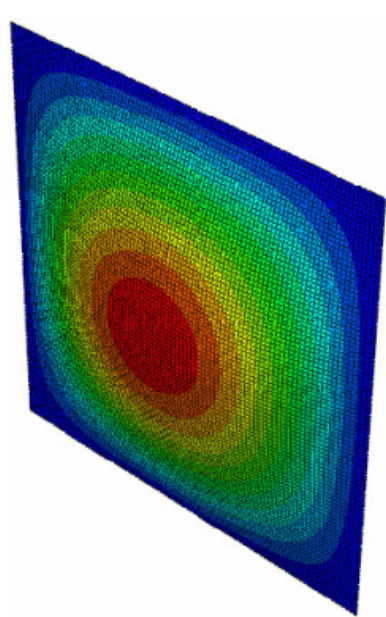

(a)

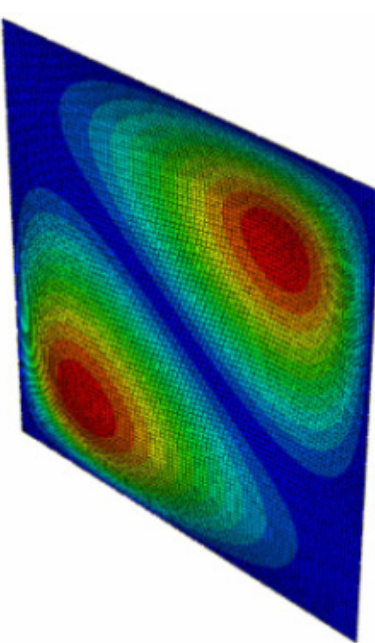

(b)
Fig. 6. Different mode shapes of a simply supported laminated composite plate with $[0 / 90 / 0 / 90 / 0]_{\mathrm{S}}$ layer sequence with $\mathrm{Al}$ layers in $(1,10)$ plies in ABAQUS. (a) Mode 1: $\bar{\omega}=4.1498$. (b) Mode 2: $\bar{\omega}=16.5007$.

was an important factor on the overall and local dynamic behaviors of these structures. It can be also seen that the dynamic response of these structures was so complicated and depended on what the plate's aspect ratio and fibers' orientation were.

\section{References}

[1] A. Asundi, A.Y.N. Choi, Fiber metal laminates: an advanced material for future aircraft, Mater. Process. Technol. 63 (1997) 384-93
[2] A. Vlot, L.B. Vogelesang, De Vries, Towards application of fiber metal laminates in large aircraft, Aircraft Eng. Aerospace Technol. 71 (1999) 558-70

[3] G. Marsh, Airframes exploit composites in battle for supremacy, Reinforced Plastics 49 (2005) 26-32

[4] D. Ngo-Cong, N. Mai-Duy, W. Karunasena, T. TranCong, Free vibration analysis of laminated composite plates based on FSDT using one-dimensional IRBFN method, Comput. Struct. 85 (2011) 1-13

[5] Y.F. Xing, B. Liu, New exact solution for free vibrations of orthotropic rectangular plates, Compos. Struct. 89 (2009) 567-74

[6] M. Ganapathi, A. Kalyani, B. Mondal, T. Prakash, Free vibration analysis of simply supported composite laminated panels, Compos. Struct. 90 (2009) 100-03

[7] A.W. Leissa, A.F. Martin, Vibration and buckling of rectangular composite plates with variable fiber spacing, Compos. Struct. 14 (1990) 339-57

[8] Y. Xiang, J.N. Reddy, Natural vibration of rectangular plates with an internal line hing using the first order shear deformation plate theory, Sound Vib. 263 (2003) 285-97

[9] J.N. Reddy, T. Kuppusamy, Natural vibrations of laminated anisotropic plates, Sound Vib. 94 (1984) 63-69

[10] H.T. Thai, S.E. Kim, Free vibration of laminated composite plates using two variable refined plate theory, Mech. Sci. 52 (2010) 626-33

[11] M.R. Aagaah, M. Mahinfalah, G.N. Jazar, Natural frequency of laminated composite plates using third order shear deformation theory, Compos. Struct. 72 (2006) 273-9

[12] K. Swaminathan, S. Patil, Analytical solutions using a higher order refined computational model with 12 degrees of freedom for the free vibration analysis of antisymmetric angle-ply plates, Compos. Struct. 82 (2008) 209-16

[13] I. Mishara, Sh. Kumar Sahu, An experimental approach to free vibration response of woven fiber composite plates under free-free boundary condition, Adv. Tech. Civil Eng. 1 (2012) 67-72

[14] K.M. Dutt, H.K. Shivanand, An experimental approach to free vibration response of carbon composite laminates, Adv. Eng. Appli. (2011) 66-68 
[15] S.Y. Lee, T. Park, Free vibration of laminated composite skew plates with central cutout, Struct. Eng. Mech. 31 (2009) 587-683

[16] Ö. Civalek, Free vibration analysis of symmetrically laminated composite plates with first-order shear deformation theory (FSDT) by discrete singular convolution method, Finite Elements in Analysis and Design 44 (2008) 725-31

[17] H.T. Thai, S.E. Kim, Free vibration of laminated composite plates using two variable refined plate theory, Mech. Sci. 52 (2010) 626-33

[18] S. Hatami, M. Azhari, M.M. Saadatpour, Free vibration of moving laminated composite plates, Compos. Struct. 80 (2007) 609-20

[19] S. Xiang, K.M. Wang, Free vibration analysis of symmetric laminated composite plates by trigonometric shear deformation theory and inverse multiquadric RBF, ThinWalled Struct. 47 (2009) 304-10

[20] U. Topal, Ü. Uzman, Free vibration analysis of laminated plates using first-order shear deformation theory, Springer Proceedings in Physics 111 (2007) 493-98

[21] W. Zhen, C. Wanji, Free vibration of laminated composite and sandwich plates using global-local higher-order theory, Sound Vib. 298 (2006) 333-49

[22] J.M. Withney, N.J. Pagano, Shear deformation in heterogeneous anisotropic plates, Appl. Mech. 37 (1970) 1031-36

[23] R.D. Mindlin, Influence of rotary inertia and shear on flexural motions of isotropic elastic plates, Appl. Mech. 18 (1951) 31-38
[24] A.P. Christoforou, S.R. Swanson, Analysis of impact response in composite plates, Solids Struct. 27 (1991) 16170

[25] S.M.R. Khalili, A. Shokuhfar, F. Ashenai Ghasemi, Effect of smart stiffening procedure on low-velocity impact response of smart structures, Mater. Process. Technol. 190 (2007) $142-52$

[26] A. Carvalho, C.G. Soares, Dynamic response of rectangular plates of composite materials subjected to impact loads, Compos. Struct. 34 (1996) 55-63

[27] K.M. Liew, Vibration of symmetrically laminated cantilever trapezoidal composite plates, Mech. Sci. 34 (1992) 299-308

[28] W.O. Wong, The effects of distributed mass loading on plate vibration behavior, Sound Vib. 252 (2002) 577-83

[29] L. Meirovitch, Fundamental of Vibrations, McGraw-Hill, New York, 2001

[30] R.H. Bhat, Natural frequencies of rectangular plates using characteristic orthogonal polynomials in RayleighRitz method, Sound Vib. 102 (1985) 493-99

[31] A.P. Christoforou, A.S. Yigit, Characterization of impact in composite plates, Compos. Struct. 43 (1998) 15-24

[32] G.H. Payeganeh, F. Ashenai Ghasemi, K. Malekzadeh, Dynamic response of fiber-metal laminates (FMLs) subjected to low-velocity impact, Thin-Walled Struct. 48 (2010) 62-70 